26. Osma, Beatriz Garcia., (2008), "Board Independence and Real Earnings Management: The Case of R\&amp; D Expenditure", Corporate Governance: An International Review, 16 (2): $116-31$.

27. Pant M., Pattanayak M., (2007), "Insider ownership and firm value: evidence from Indian corporate sector", Economic and Political Weekly. - P. 1459-1467.

28. Pi, L. and S.G. Timme, (1993) "Corporate Control and Bank Efficiency”, Journal of Banking and Finance, 17, pp. 515-530.

29. Richardson S., (2006), "Over-investment of free cash flow”, Review of Account Studies, 11, 159-189.

30. Selarka E., (2005), "Ownership concentration and firm value: A study from the Indian corporate sector”, Emerging Markets Finance and Trade. - Vol. 41. - №. 6. - P. 83-108.

\title{
«ЭФФЕКТ РАЗМЕРА» \\ И ЗАТРАТЫ НА СОБСТВЕННЫЙ КАПИТАЛ
}

Фомкина Софья Анатольевна ${ }^{1}$

\begin{abstract}
«Эффект размера» до сих пор остается одной из загадок рынков капитала. Данный эффект впервые был обнаружен Банзом [Banz, 1981] при тестировании модели ценообразования активов (САРМ) на американском рынке. Данное открытие повлекло дальнейшее исследование этой закономерности на других развитых и развивающихся рынках капитала. Однако до сих пор в научной среде нет единого мнения о действительном существовании данного эффекта на рынках капитала и его размере. Вместе с тем премия за малую капитализацию активно используется в практической деятельности компаний, фондов и отдельных аналитиков в процессе оценки затрат на собственный капитал.

В работе представлен обзор исследований, посвященных анализу данного эффекта на развитых и развивающихся рынках капитала. Систематизированы и обобщены различные подходы к оценке влияния размера компании на величину затрат на собственный капитал, сопоставлены предлагаемые авторами прокси размера, проведен анализ полученных эмпирических результатов. Обсуждаются особенности, выявленные в рамках анализа эффекта размера, и причины их возникновения, а также появления наблюдаемого эффекта в целом на рынках капитала и его дальнейшего исчезновения в ряде стран.
\end{abstract}

Ключевые слова: эффект размера, премия за размер, затраты на собственный капитал, рынки капитала

JEL: G12, G15, G32

\section{Открытие «эффекта размера» и его эволюция в исследованиях на развитых рынках капитала}

Предпосылки модели ценообразования активов (САРМ) не раз подвергались критике. Многие авторы [Banz, 1981; Reinganum, 1981; Fama, French, 1992; Fama, French, 1993] отмечают недостаточность объясняющей силы рыночной премии в динамике доходностей акций при использовании реальных данных рынков капитала.

Сложившаяся ситуация послужила началом дальнейших исследований и попыток усовершенствования модели САРМ. В последние несколько десятилетий разработаны и предложены новые способы оценки рисков и соответствующие модели определения затрат на собственный капитал. Тем не менее тестирование данных моделей приводит к противоречивым результатам.

«Эффект размера» (size effect) впервые был обнаружен Банзом [Banz, 1981] при тестировании модели ценообразования активов (САРM) на американском рынке. Согласно данному иссле-

${ }^{1}$ Аспирант, факультет экономических наук, Департамент финансов, НИУ ВШЭ. 
дованию размер компании оказывает влияние на величину затрат на собственный капитал, а именно акции наименьших по размеру компаний показывают наибольшие доходности по сравнению с крупными компаниями. Данное открытие повлекло дальнейшее исследование этой закономерности на других развитых и развивающихся рынках капитала, однако систематическое наличие указанного эффекта не было подтверждено. Ряд исследователей отмечают наличие эффекта размера (таблицы $1,2,4,5$ ), другие исключают его [Fama and French, 1992Chan et al., 2000; Horowitz et al., 2000a; Horowitz et al., 2000b; Amihud, 2002; Michou et al., 2010; Fama and French, 2012; Ивашковская и др., 2012].

Многие работы, посвященные «эффекту размера», основаны на информации о компаниях, торгующихся на американских фондовых площадках (таблица 1). Согласно данным исследованиям, премия за размер в США варьировалась в диапазоне от 0,4\% [Banz, 1981] до 2,52\% [Keim, 1993]. Однако исследования более поздних периодов показывают снижение величины премии за размер и ее значимости, согласно им с 1980-х гг. данный эффект уменьшился или вовсе исчез [Fama and French, 1992; Chan et al., 2000; Horowitz et al., 2000a; Horowitz et al., 2000b; Amihud, 2002; Fama and French, 2012]. Некоторые авторы [Horowitz et al., 2000b] настаивают на том, что обнаруженный в других работах «эффект размера» является лишь академической находкой.

Ряд авторов [Lo and MacKinlay, 1990; Black, 1993] обращают внимание на то, что в значительном числе исследований используются одинаковые выборки (бумаги и периоды), чтобы обнаружить «эффект размера» и другие аномалии ценообразования активов. При этом только самые успешные, необычные и поразительные результаты публикуются.

Таблица 1

Результаты некоторых исследований эффекта размера на рынке США

\begin{tabular}{ccccc} 
Исследование & $\begin{array}{c}\text { Количество } \\
\text { портфелей }\end{array}$ & Выборка & Период исследования & $\begin{array}{c}\text { Премия за размер, \% в } \\
\text { месяц }\end{array}$ \\
\hline Banz [1981] & 5 & NYSE, AMEX & $1936-1977$ & 0,4 \\
\hline Reinganum [1981] & 10 & 566 & $1975-1979$ & 1,77 \\
Keim [1993] & 10 & $1500-2400$ & $1963-1979$ & 2,52 \\
\hline Fama and French [1993] & 10 & NYSE, AMEX \& NASDAQ & $1962-1989$ & 1970 \\
\hline Al-Rjoub et al. [2005] & 10 & NYSE, AMEX \& NASDAQ & $1970-1999$ & 1,01 \\
\hline Easterday et al. [2009] & 10 & NYSE, AMEX \& NASDAQ & $1946-2007$ & 0,5 \\
\hline
\end{tabular}

«Эффект размера», согласно исследованиям для других развитых рынков капитала, колеблется в зависимости от выбранного рынка капитала, а также внутри одного рынка в зависимости от выбранного периода исследования. Результаты данных исследований (таблица 2) приводят к тому, что при оценке затрат на собственный капитал на одном из рынков капитала использование премий за размер других рынков может привести к некорректным результатам.

Таблийа 2

Результаты некоторых исследований эффекта размера на развитых рынках

\begin{tabular}{cccc} 
Исследование & Страна & Период & $\begin{array}{c}\text { Премия за размер в } \\
\text { месяц, \% }\end{array}$ \\
\hline Beedles [1992] & Австралия & $1974-1987$ & 5,06 (в год) \\
Mills and Jordanov [2000] & Великобритания & $1985-1995$ & 2,14 \\
Leledakis et al. [2003] & Греция & $1990-2000$ & 1,39 \\
Annaert et al. [2002] & Европейские страны & $1974-2000$ & 1,45 \\
Elfakhani et al. [1998] & Канада & $1979-1992$ & 0,98 \\
Doeswijk [1997] & Нидерланды & $1973-1995$ & 1,6 (в год) \\
Gillan [1990] & Новая Зеландия & $1977-1984$ & 0,51 \\
Wong et al. [1990] & Сингапур & $1975-1985$ & 0,41 \\
Garza-Gomez et al. [1998] & Япония & $1957-1994$ & 1,27 \\
\hline
\end{tabular}


Наиболее распространенная теория, объясняющая рациональные причины наличия «эффекта размера» на рынке, заключается в том, что инвесторы требуют большей доходности для маленьких компаний в связи с тем, что они характеризуются более высоким риском. Это может быть связано с:

- Необходимостью компенсации за риск [Cho, 2012]: малые по размеру компании чаще имеют проблемы с денежным потоком, имеют более высокую вероятность выхода в дефолт по кредитам, более высокий уровень риска в периоды рецессии.

- Проблемой ликвидности: небольшие компании менее ликвидны, и инвесторы требуют получение большей доходности [Amihud, Mendelson, 1986; Hearn et al., 2010; Chen et al., 2010]. При этом аналитики при расчете беты таких компаний могут столкнуться с недостатком информации для получения корректных оценок: поскольку компании менее ликвидны, то торги по их акциям могут осуществляться не ежедневно.

- Информационной асимметрией: небольшие компании менее диверсифицированы и обладают меньшим объемом доступной для рынка информации, в связи с чем инвесторы также требуют получения более высокой доходности [Zhang, 2006; Apergis, Payne, 2014].

Следует отметить, что, как правило, «эффект размера» наблюдается в группах, где представлены только самые малые по размеру компании, и является незначимым при сравнении средних и больших компаний.

Как ранее отмечалось, вместе с тем авторы значительного числа исследований показали, что с 1980-х гг. данный эффект уменьшается в США, но также и на других развитых рынках капитала, в том числе и в Великобритании [Dimson and Marsh, 1999; Michou et al., 2010]. Димсон и Марш [Dimson and Marsh, 1999] показали, что эта рыночная аномалия, возможно, исчезла или же обрела обратный эффект - премию за размер для крупных компаний.

Фама и Френч [Fama and French, 2012] также не смогли найти премию за размер в 23 развитых странах в течение более 20 лет (период: ноябрь 1989 г. - март 2011 г.).

- Исчезновение «эффекта размера» авторы в основном связывают с:

- публикацией статей об эффекте размера [Horowitz et al., 2000a[, после чего стали расти цены на акции маленьких компаний, тем самым уменьшая доходность;

- пассивным инвестированием в индексные взаимные фонды [Horowitz et al., 2000a], специализирующиеся на вложениях в малые по размерам капитализации компании [Horowitz et al., 2000a; Dimson and Marsh, 1999];

- изменениями в рыночной ликвидности [Amihud, 2002];

• поведенческой теорией [Lemmon, Portniaguina, 2006], утверждающей, что рост потребительского доверия (прокси настроения инвестора) ведет к увеличению спроса на акции небольших компаний.

Одним из последствий подобных результатов является признание того, что размер не является наилучшим прокси для риска, так как имеет тенденцию к изменению во времени.

\section{Прокси размера}

В качестве прокси размера в большинстве случаев исследователи используют рыночную капитализацию, однако также существуют и другие альтернативы, в том числе балансовые показатели компании (таблица 3).

Таблииа 3

Варианты прокси размера

\begin{tabular}{|c|c|}
\hline Прокси размера компании & Автор (год) \\
\hline Enterprise value & Sehgal, Tripathi [2005] \\
\hline Market value of common equity & Bce paccмотренные отчеты и статьи \\
\hline
\end{tabular}




\begin{tabular}{|c|c|}
\hline Прокси размера компании & Автор (год) \\
\hline Book value of common equity & Duff \& Phelps Risk Premium Report \\
\hline Market value of invested capital & Duff \& Phelps Risk Premium Report \\
Total Assets & Duff \& Phelps Risk Premium Report, Berk [1996], \\
Sehgal, Tripathi [2005]
\end{tabular}

Согласно авторам отчета Duff \& Phelps Risk Premium Report, существуют две основные причины для поиска альтернативных оценок размера:

- Классификация компаний по рыночной стоимости может привести к смещению результатов, так как рыночная стоимость является функцией не только размера компании, но и ставки дисконтирования. Некоторые компании могут иметь низкую рыночную стоимость по причине их высокой рискованности, что соответствует более высокой ставке дисконтирования.

- Компании могут иметь низкую рыночную стоимость акций при больших объемах продаж или большой операционной прибыли и одновременно высоком значении рычага. Следовательно, рыночная стоимость акций не всегда является аналогом размера операционной деятельности компании.

Таким образом, использование балансовых показателей, таких как активы или чистая прибыль, позволит устранить потребность в использовании приближенных оценок размера.

Однако исследование Берк [Berk, 1996] показало неспособность четырех альтернативных (балансовых) оценок размера компании предоставить такую же объясняющую силу, как и рыночная капитализация.

\section{Методология исследования эффекта размера}

В научной литературе наиболее распространены два подхода: регрессионный анализ и сравнение доходностей портфелей, сформированных на основе показателя размера компании.

Сравнение доходностей портфелей, сформированных на основе показателя размера, проводится за определенные временные интервалы между собой и с «ожидаемой» величиной доходности [Keim, 1983; Ibbotson SBBI Classic Yearbook; Duff \& Phelps Risk Premium Report]. По мнению Фамы и Френча [Fama and French, 1992]. такой поход позволяет получить «простую картину» (simple picture), с помощью которой можно увидеть, как средняя доходность варьируется в зависимости от размера компаний.

Регрессионный анализ предполагает построение зависимости доходности компании от прокси размера компании и других переменных (показатели ликвидности, book-to-market и т.д.). В большинстве случаев для оценки применяется методология Фамы-Макбет [Fama and Macbeth, 1973]. При этом общая выборка компаний делится на подгруппы, для каждой из них оценивается регрессия (временной ряд) и сравниваются коэффициенты при независимых переменных, оцененные для подгрупп [Amihud, 2002]. Затем строится перекрестная регрессия (cross-sectional) или регрессия на основе панельных данных, где в качестве регрессоров выступают коэффициенты, полученные после оценки модели временных рядов. Результаты текущей оценки показывают чувствительность доходности компании к ее размеру (особое внимание уделяется знаку коэффициента при регрессоре) и степени объясняющей силы модели. При этом следует отметить, что в некоторых случаях анализ проводится для моделей с 
добавлением абсолютного показателя размера (а также дополнительных факторов) [Amihud and Mendelson, 1986; Amihud, 2002]. Некоторые авторы и вовсе опускают рыночный риск, как объясняющую переменную [Berk, 1996; Horowitz et al., 2000a; Horowitz et al., 2000b; Easterday et al., 2009].

В этом подходе анализа отдельно следует выделить модель Фамы-Френча [Fama and French, 1992; Fama and French, 1993) и ее модификации [Zhang, 2006; Khan et al., 2012]. Трехфакторная модель Фамы-Френча [Fama and French, 1992; Fama and French, 1993] была создана на волне появившейся критики модели, предложенной Шарпом, Линтнером и Блеком [Sharpe, 1964; Lintner ,1965; Black et al., 1972], согласно которой бета портфеля может объяснить изменчивость его доходности. Однако другие исследователи [Reinganum, 1981; Lakonishok and Shapiro, 1986] выявили, что с начала 1960-х гг. этот факт не подтверждается. Фама и Френч [Fama and French, 1992; Fama and French, 1993] предложили улучшить модель и добавить в нее показатели размера и возможностей роста компании. На данных 1963-1990 гг. результат был поразительным - объясняющая сила модели составила около 99\%, но бета портфеля не объясняла динамику доходности. После публикации этой модели другие исследователи проверяли ее работоспособность. Часть авторов подвергли критике модель и опровергли ее результаты [Daniel and Titman, 1997], другие же, наоборот, подтвердили ее эффективность [Davis et al., 2000].

Многие исследователи делают выводы на основе нескольких подходов одновременно [Banz, 1981; Horowitz et al., 2000a; Horowitz et al., 2000b; Easterday et al., 2009; Michou et al., 2010].

\section{Модели оценки премии за размер в отчетах, публикуемых на ежегодной основе}

В настоящее время на рынке представлен отчет Valuation Handbook - Guide to Cost of Capital, который предоставляет данные, ранее публикуемые Morningstar / Ibbotson SBBI Valuation Yearbook (публикация прекращена с 2013 г.) и Duff \& Phelps Risk Premium Report (более не публикуется как отдельное издание). Тем не менее в данной работе мы остановимся на основных особенностях отчетов Ibbotson SBBI Classic Yearbook и Duff \& Phelps Risk Premium Report, данными которых пользовались в течение многих лет консультанты, аналитики, брокеры и другие специалисты.

Ibbotson SBBI Classic Yearbook (далее - Ibbotson Yearbook) - это издание, ранее ежегодно публикуемое Morningstar, Inc., которое представляет собой исследование данных рынка капитала в Соединенных Штатах. Материалы этой книги (сейчас Valuation Handbook - Guide to Cost of Capital) используются для анализа доходности активов. Издание содержит информацию начиная с 1926 г. о доходности индексов, крупных и малых компаний, государственных ценных бумаг и другую информацию об американском рынке капитала.

Для некоторых частей Ibbotson Yearbook ранее существовал аналог - Duff \& Phelps Risk Premium Report (далеe - Duff \& Phelps Report), который публиковался ежегодно с 1996 г. и использовал данные рынка капитала США с 1963 г.

Один из самых популярных разделов данных отчетов - это раздел, в котором представлены премии за малую капитализацию.

Согласно Ibbotson Yearbook, методология по оценке премии за малую капитализацию представляет собой разбиение выборки (данные NYSE / AMEX / NASDAQ) на 10 портфелей, эквивалентных по числу компаний в каждом, на основе показателя размера компании - рыночной капитализации. Портфели формируются ежеквартально. Для каждого из портфелей рассчитывается доходность за каждый месяц как средневзвешенная доходностей индивидуальных активов, входящих в портфель, после этого оценивается бета-коэффициент на основе рыночной модели (market model). Полученный коэффициент является основой для получения «расчетной» доходности, которая сравнивается с доходностью, полученной в действительности. Разница между данными показателями и является премией за малую капитализацию. Она 
рассчитывается для всех 10 портфелей, а также для групп портфелей средних компаний (куда входят портфели с 3-го по 5-й), малых компаний (6-8-й портфели) и микрокомпаний (9-10-й портфели). Параметры, используемые для оценки бета-коэффициента в Ibbotson Yearbook, являются следующими:

- Безрисковая ставка - доходность 30-day U.S. Treasury bill total return (среднее значение.

- Доходность рыночного портфеля - доходность индекса S\&P500. Дополнительно рассматривается альтернатива, где в качестве рыночной доходности берется средневзвешенная доходность по компаниям NYSE. Размер премии за размер практически не меняется.

Duff \& Phelps Report для оценки премии за малую капитализацию использует методологию, в значительной степени схожую с методологией Ibbotson. Основные же различия между ними заключались в следующем:

- разбиение осуществляется на 25 портфелей, а не на 10;

- используются альтернативные показатели размера (таблица 3);

- выборка включает доходности акций с 1963 г., а не с 1926 г.

Следует отметить, что данные из отчетов применяются не только для оценки активов, расположенных в Соединенных Штатах, но также и для других развитых и развивающихся рынков капитала.

\section{Особенности исследований «эффекта размера»}

Рассматривая методологию анализа «эффекта размера», следует уделить внимание ряду особенностей, с которыми столкнулись исследователи.

В первую очередь, была выявлена связь между «эффектом размера» с показателями ликвидности. Как говорилось ранее, одна из причин «эффекта размера»- риск ликвидности. Обычно акции малых компании наименее ликвидные. Тогда возможно ли, что показатель ликвидности может заменить прокси размера в модели?

В ряде работ на данную тему [Amihud and Mendelson, 1986; Brennan and Subrahmanyam, 1996] поднимается вопрос о том, что ликвидность может быть важным фактором, оказывающим влияния на доходность активов. Бекарт и соавторы [Bekaert et al., 2007] утверждают, что риск ликвидности является особенно важным для менее сложных рынков, где количество ценных бумаг невелико и инвесторов не хватает. Большинство исследователей подтверждают наличие связи между показателем ликвидности и доходностью акций компаний как на развитых, так и на развивающихся рынках капитала [Pastor and, Stambaugh, 2003; Bekaert et al., 2007; Hearn et al., 2010; Lee, 2011; Liang, 2012].

Кроме ликвидности, многие исследователи выделяют такую особенность «эффекта размера», как сезонность. На рынке США было выявлено, что наибольшие доходности по акциям наблюдаются в январе и далее уменьшаются или вовсе становятся отрицательными [Horowitz et al., 2000a]. При этом подобное явление в большей степени проявляется для компаний с малой капитализацией [Keim, 1983; Reinganum, 1983; Easterday et al., 2009].

Некоторые исследователи предполагают, что причиной этого являются попытки инвесторов зафиксировать убыток в декабре и получить налоговую экономию [Keim, 1983]. С другой стороны, в некоторых странах в конце года в компаниях выплачивают бонусы, увеличивающие склонность к повышенному риску, что может привести к росту спроса на высокорисковые бумаги, в том числе и акции компаний с малой капитализацией [Wong et al., 1990; Tong, 1992; Chen and Chien, 2011]. 


\section{Исследования «эффекта размера» \\ на развивающихся рынках капитала}

Исследований, посвященных анализу премии за размер на развивающихся рынках капитала, в том числе и в России, значительно меньшее количество (таблица 4). Согласно им, величина премии за размер на развивающихся рынках колеблется от $0,83 \%$ для рынка Китая за период 1991-2004 гг. [Eun and Huang, 2007] до 4,16\% для рынка Мексики за период 1988-1992 гг. [Herrera and Lockwood, 1994].

Таблица 4

Результаты исследований эффекта размера на развивающихся рынках

\begin{tabular}{cccc} 
Исследование & Страна & Период & Премия за размер в месяц, \% \\
\hline Herrera, Lockwood [1994] & Мексика & $1987-1992$ & 4,16 \\
Aksu, Onder [2003] & Турция & $1993-1997$ & 3,42 \\
Eun, Huang [2007] & Китай & $1991-2004$ & 0,83 \\
Singh [2009] & Индия & $1991-2002$ & 1,72 \\
Wu [2010] & Китай & $1992-2009$ & 2,23 \\
\hline
\end{tabular}

Значительная группа исследований на развивающихся рынках капитала посвящена тестированию модели Фамы-Френча [Fama and French, 1992, 1993]. Согласно большинству из них, данная модель достаточно хорошо объясняет доходности акций компаний по сравнению с традиционной САРМ и свидетельствует о существовании влияния размера компании на требуемую доходность. Аль-Мвалла и Карашех [Al-Mwalla and Karasneh, 2011] показали, что для всех рассмотренных портфелей компаний Иордании с 1999 г. по 2010 г. объясняющая способность модели выше, чем у САРМ, и премия за размер составляет $0,35 \%$ в месяц. Дрю и Вирарагаван [Drew and Veeraraghavan, 2002] показали, что для компаний Малайзии с 1991 г. по 1999 г. трехфакторная модель лучше объясняет динамику доходностей (R2 = $0,95)$, и премия (фактор SMB) составила 1,48\%. В свою очередь, для китайского рынка Дрю и Вирарагаван [Drew et al., 2003] получили премию (фактор SMB) в размере 0,93\%. В рамках исследования рынка Пакистана [Mirza and Shahid, 2008; Hassan and Javed, 2011] показали различные премии за размер для периода 2003-2007 гг., что связано с разным количеством компаний в выборке.

Таблица 5

Результаты некоторых исследований эффекта размера на развивающихся рынках в рамках трехфакторной модели Фамы-Френча

\begin{tabular}{|c|c|c|c|}
\hline Исследование & Выборка & Период & Премия SMB, месячная? \% \\
\hline Drew and Veeraraghavan [2002] & Малайзия & 1992-1999 & 1,48 \\
\hline Drew et al. [2003] & Китай (SSE) & $1993-2001$ & 0,93 (значима для $\mathrm{S}$ ) \\
\hline \multirow{3}{*}{ Shum, Tang [2005] } & Гонконг (HKSE), & \multirow{3}{*}{ 1986-1998 } & 1,42 \\
\hline & Тайвань (TWSE), & & 1,32 \\
\hline & Сингапур (SES) & & 1,75 \\
\hline Bundoo [2008] & Маврикий (SEM) & 1998-2004 & 0,69 \\
\hline Hassan and Javed [2011] & $\begin{array}{c}\text { Пакистан, } \\
275 \text { компаний }\end{array}$ & $2000-2007$ & -0,0004 (дневная) \\
\hline Al-Mwalla and Karasneh [2011] & Иордания (ASE) & $\begin{array}{c}\text { июнь } 1999 \text { - июнь } \\
2010\end{array}$ & 0,35 \\
\hline
\end{tabular}

Тестирование модели Фамы-Френча на российских данных было проведено в рамках работы, представленной в монографии Ивашковской и соавторов [2012], результаты которой также подтверждают наличие эффекта размера. Однако величина данного эффекта весьма нестабильна. 
В целом следует отметить то, что в большинстве работ отмечена более высокая объясняющая способность трехфакторной модели относительно САРМ и наблюдается положительная премия за размер. Исследователями этот феномен объясняется тем, что для малых компаний уровень риска выше и не может быть объяснен в рамках САРМ.

\section{Заключение}

В последние несколько десятилетий разработаны и предложены новые способы оценки рисков и соответствующие модели определения затрат на собственный капитал. Тем не менее тестирование данных моделей приводит к противоречивым результатам. Большое количество исследований подтверждает существование премии за размер в доходности акций, одновременно другая - отрицает существование данного эффекта.

Вместе с тем, практикующие специалисты игнорируют результаты исследований, где сообщается о различных величинах премии за размер в разрезе стран и периодов, постепенном исчезновении «эффекта размера» или изменении его знака и продолжают опираться на мнение, что акции компаний с малой капитализацией связаны не только с большим риском, но и большей доходностью. Многие из них при оценке затрат на собственный капитал используют данные о премиях в отчетах, публикуемых на постоянной основе и опирающихся на анализ американского рынка капитала, и не задумываются о том, что в случае применения данных отчетов для развивающихся рынков капитала могут быть получены некорректные оценки.

Таким образом, вопрос о влиянии размера компании на доходность ее акций остается открытым, исследователи не пришли к единой точке зрения, в связи с чем данное направление исследований остается весьма актуальным, в особенности для развивающихся рынков капитала.

\section{Список литературы}

1. Ивашковская И.В., Григорьева С.А., Кокорева М.С., Степанова А.Н. и др. Корпоративные финансовые решения. Эмпирический анализ российских компаний (корпоративные финансовые решения на развивающихся рынках капитала): Монография / Под науч. ред. д-ра экон. наук И.В. Ивашковской. М: ИНФРА-М, 2012. - 281 с.

2. Aksu M.H. and Onder T. (2003) The Size and Book-to-Market Effects and Their Role as Risk Proxies in the Istanbul Stock Exchange. SSRN Working Paper. Available at: http://ssrn. com/abstract $=250919$ (accessed 30 September 2016).

3. Al-Mwalla M., Karasneh M. (2011) Fama \& French Three Factor Model: Evidence from Emerging Market. European Journal of Economics, Finance and Administrative Sciences, no. 41, pp. 132-141. Available at: http://s3.amazonaws.com/academia.edu. documents/32657095.

4. Al-Rjoub S.A.M., Varela O. and Hassan M.K. (2005) The size effect reversal in the USA. Applied Financial Economics, vol. 15, pp. 1189-1197. doi:10.1080/09603100500359542.

5. Amihud Y. (2002) Illiquidity and stock returns: cross-section and time-series effects. Journal of Financial Markets, vol. 5, no.1, pp. 31-56. doi:10.1016/S1386-4181(01)00024-6.

6. Amihud Y. and Mendelson H. (1986) Asset pricing and the bid-ask spread. Journal of Financial Economics, vol. 17, pp. 223-249. doi:10.1016/0304-405X(86)90065-6.

7. Annaert J., van Holle F., Crombez J. and Spinel B. (2002) Value and size effect: Now you see it, now you don't. SSRN Working Paper. Available at: http://ssrn.com/abstract=950899 (accessed 30 September 2016).

8. Apergis N. and Payne J.E. (2014) Resurrecting the size effect Evidence from a panel nonlinear cointegration model for the G7 stock markets. Review of Financial economics vol. 23, no.1, pp. 46-53. doi:10.1016/j.rfe.2013.08.003.

9. Banz R.W. (1981) The relationship between return and market value of common stocks. Journal of Financial Economics, vol. 9, no.1, pp. 3-18. doi:10.1016/0304-405X(81)90018-0. 
10. Beedles W.L. (1992) Small firm equity cost: evidence from Australia. Journal of Small Business Management, vol. 30, pp. 57-65. Available at: http://crawl.prod.proquest. com.s3.amazonaws.com/fpcache/f00012e0d24400454a4ff2505169771a.pdf?AWSAccessK eyId=AKIAJF7V7KNV2KKY2NUQ\&Expires $=1475427763 \&$ Signature $=$ KFXVBLkr\%2F gSqA1z4oPeri94cLKc\%3D (accessed 30 September 2016).

11. Bekaert G., Harvey C.R. and Lundbland C. (2007) Liquidity and expected returns: lessons from emerging markets. Review of Financial Studies, vol. 20, no6, pp. 1783-1831. doi:10.1093/rfs/hhm030

12. Berk B. (1996) An Empirical Re-examination of the Relation Between Firm Size and Return. Revised Working Paper 93-BJ-001 (1996), Seattle: School of Business Administration, University of Washington. Available at: http://citeseerx.ist.psu.edu/viewdoc/download? doi=10.1.1.195.7084\&rep=rep1\&type=pdf (accessed 30 September 2016).

13. Black F., Jensen M.C. and Scholes M. (1972) The Capital Asset Pricing Model: Some Empirical Tests. SSRN Working Paper. Available at: http://ssrn.com/abstract=908569 (accessed 30 September 2016).

14. Brennan M.J. and Subrahmanyam A. (1996) Market microstructure and asset pricing: On the compensation for illiquidity in stock returns. Journal of Financial Economics, vol. 41, no. 3, pp. 441-464. doi:10.1016/0304-405X(95)00870-K.

15. Bundoo S.K. (2008) An Augmented Fama and French Three-Factor Model: New Evidence From An Emerging Stock Market. Applied Economics Letters, vol. 15, pp. 1213-1218. doi:10.1080/13504850601018049.

16. Chan L.K.C., Karceski J. and Lakonishok J. (2000) New paradigm or same old hype in equity investing? Financial Analysts Journal, vol. 56, no. 4, pp. 23-36. doi:10.2469/faj.v56. n4.2371.

17. Chen T.S. and Chien C.C. (2011) Size effect in January and cultural influences in an emerging stock market: The perspective of behavioral finance. Pacific-Basin Finance Journal, vol. 19, no. 2, pp. 208-229. doi:10.1016/j.pacfin.2010.10.002.

18. Chen Z., Ibbotson R.G. and Hu W.Y. (2010) Liquidity as an investment style. SSRN Working Paper. Available at: http://ssrn.com/abstract=1817889 (accessed 30 September 2016).

19. Cho S. (2012) The Size Premium: What Role Does Macroeconomic Risk Play? SSRN Working Paper. Available at: http://ssrn.com/abstract=1969828 (accessed 30 September 2016).

20. Daniel K. and Titman S. (1997) Evidence on the Characteristics of Cross Sectional variation in stock returns. Journal of Finance, vol. 52, no. 1, pp. 1-33. doi:10.1111/j.1540-6261. 1997.tb03806.x.

21. Dimson E. and Marsh P. (1999) Murphy's law and market anomalies. Journal of Portfolio Management, vol. 25, no. 2, 1999, pp. 53-69. Available at: http://ssrn.com/abstract $=137337$ (accessed 30 September 2016).

22. Doeswijk R.Q. (1997) Contrarian Investment in the Dutch Stock Market. De Economist, vol. 145, no. 4, pp. 573-598. doi:10.1023/A:1003133310376.

23. Drew M.E. and Veeraraghavan M. (2002) A closer look at the size and value premium in emerging markets: evidence from the Kuala Lumpur stock exchange. Asian Economic Journal, vol. 16, no. 4, pp. 337-351. doi:10.1111/1467-8381.00156.

24. Drew M.E. (2003) Beta, Firm Size, Book-to-Market Equity and Stock Returns: Further Evidence from Emerging Markets. Journal of the Asian Pacific Economy, vol. 8, no. 3, pp. 354-379. doi:10.1080/13547860306289.

25. Drew M.E., Naughton T. and Veeraraghavan M. (2003) Firm size, book-to-market equity and security returns: evidence from the Shanghai Stock Exchange. Australian Journal of Management, vol. 28, no. 2, pp. 119-139. doi:10.1177/031289620302800201. 
26. Easterday K.E., Sen P.K. and Stephan J.A. (2009) The persistence of the small firm/January effect: Is it consistent with investors' learning and arbitrage efforts? The Quarterly Review of Economics and Finance, vol. 49, no. 3, pp. 1172-1193. doi:10.1016/j.qref.2008.07.001.

27. Elfakhani S., Lockwood L.J. and Zaher T.S. (1998) Small Firm and Value Effects in the Canadian Stock Market. Journal of Financial Research, vol. 21, no. 3, pp. 277-292. doi:10.1111/j.1475-6803.1998.tb00686.x.

28. Eun C.S. and Huang W. (2007) Asset pricing in China's domestic stock markets: Is there a logic? Pacific-Basin Finance Journal, vol. 15, no. 5, pp. 52-480. doi:10.1016/j.pacfin. 2006.11.002

29. Fama E.F. and French K.R. (1992) The Cross-Section of Expected Stock Returns. The Journal of Finance, vol. 47, no. 2, pp. 427-465. doi:10.1111/j.1540-6261.1992.tb04398.x.

30. Fama E.F. and French K.R. (1993) Common risk factors in the returns on stocks and bonds. Journal of Financial Economics, vol.33, no. 1,pp.3-56. doi:10.1016/0304-405X(93)90023-5.

31. Fama E.F., French K.R. (2012) Size, value, and momentum in international stock returns. Journal of Financial Economics, vol. 105, no 3, pp. 457-472. doi:10.1016/j.jfineco. 2012.05.011

32. Fama E.F. and MacBeth J.D. (1973) Risk, return and equilibrium: Empirical tests. Journal of Political Economy, vol. 81, no. 3, pp. 607-636. Available at: http://www.jstor.org/stable/ 1831028?seq=1\#page_scan_tab_contents (accessed 30 September 2016).

33. Garza-Gómez X., Hodoshima J. and Kunimura M. (1998) Does size really matter in Japan? Financial Analysts Journal, vol. 54, no. 6, pp. 22-34. doi:10.2469/faj.v54.n6.2222.

34. Gillan F.L. (1990) An Investigation into CAPM Anomalies in New Zealand: The Small Firm and Price-Earnings Ratio Effects. Asia Pacific Journal of Management, vol. 7, no. 2, pp. 63-78. doi:10.1007/BF01951479.

35. Grabowski R.J. (2013) Risk Premium Report. Chicago, Duff \& Phelps, LLC. Available at: $\quad$ http://www.duffandphelps.com/assets/pdfs/publications/valuation/(excerpt)\%202013 \%20duff\%20phelps\%20risk\%20premium\%20report.pdf (accessed 30 September 2016).

36. Hassan A. and Javed M.T. (2011) Size and value premium in Pakistani equity market. African Journal of Business Management, vol. 16, no. 5, pp. 6747-6755. doi:10.5897/AJBM10.817.

37. Hearn B., Piesse J. and Strange R. (2010) Market liquidity and stock size premia in emerging financial markets: the implications for foreign investment. International Business Review, vol. 19, no. 5, pp. 489-501. doi:10.1016/j.ibusrev.2009.02.009.

38. Herrera M.J. and Lockwood L.J. (1994) The Size Effect in the Mexican Stock Market. Journal of Banking and Finance, vol. 18, no. 4, pp. 621-632. doi:10.1016/0378-4266(93)00010-M/

39. Horowitz J.L., Loughran T. and Savin N.E. (2000a) The disappearing size effect. Research in Economics, vol. 54, no 1, pp. 83-100. doi:10.1006/reec.1999.0207.

40. Horowitz J.L., Loughran T., Savin N.E. (2000b) Three analyses of the firm size premium. Journal of Empirical Finance, vol. 7, no2, pp. 143-153. doi:10.1016/S0927-5398(00)000086

41. Keim D.B. (1983) Size-related anomalies and stock return seasonality: Further empirical evidence. Journal of Financial Economics, vol. 12, no. 1, pp. 13-32. doi:10.1016/0304405X(83)90025-9.

42. Karasneh M._Fama_French_Three_Factor_Model_Evidence_f.pdf?AWSAccessKeyId= AKIAJ56TQJRTW $\overline{S M T N P E A \& E x p i r e s}=14 \overline{75426899} \&$ Signature=nlQdBpYNktRiSa1NE XzYBLUvbZ4\%3D\&response-content-disposition=inline\%3B\%20filename\%3DMahmoud _Karasneh_Fama_and_French_Three_F.pdf (accessed 30 September 2016).

43. Khan F., Hassan A. and Ali S. (2012) Size, Leverage and stock returns: evidence from Pakistan. International Journal of Academic Research, vol. 4, no 1, pp. 24-32. Available at: http://s3.amazonaws.com/academia.edu.documents/34544274/SIZE__LEVERAGE_ 
AND_STOCKS_RETURNS_EVIDENCE.pdf?AWSAccessKeyId=AKIAJ56TQJRTW SMTNPEA\&Expires $=1475430530 \&$ Signature $=$ RVnosG7JD1bJ5vzFMs6RJItjZuY\%3 D\&response-content-disposition $=$ inline $\% 3 B \% 20$ filename $\% 3$ D24_PART_B._SOCIAL_ SCIENCES_AND_HUMANITIE.pdf (accessed 30 September 2016).

44. Lakonishok J. and Shapiro A.C. (1986) Systematic risk, total risk and size as determinants of stock market returns. Journal of Banking and Finance, vol. 10, no. 1, pp. 115-132. doi:10.1016/0378-4266(86)90023-3.

45. Lee K. (2011) The world price of liquidity risk. Journal of Financial Economics, vol. 99, no. 1, pp. 136-161. doi:10.1016/j.jfineco.2010.08.003.

46. Leledakis G., Davidson I. and Karathanassis G. (2003) Cross-sectional estimation of stock returns in small markets: The case of the Athens Stock Exchange. Applied Financial Economics, vol. 13, no. 6, pp. 413-426. doi:10.1080/09603100210143118.

47. Lemmon M. and Portniaguina E. (2006) Consumer confidence and asset prices: Some empirical evidence. The Review of Financial Studies, vol. 19, no. 4, pp. 1499-1529. doi:10.1093/rfs/hhj038.

48. Lintner J. (1965) The Valuation of Risk Assets and the Selection of Risky Investments in Stock Portfolios and Capital Budgets. The Review of Economics and Statistics, vol. 47, no. 1, pp. 13-37. doi:10.2307/1924119.

49. Lo A.W. and MacKinlay A.C. (1990) Data-snooping biases in tests of financial asset pricing models. Review of Financial Studies, vol. 3, no. 3, pp. 431-467. doi:10.1093/rfs/3.3.431.

50. Michou M., Mouselli S. and StarkA. (2010)Fundamental analysis and the modelling of normal returns in the UK. SSRN Working Paper. Available at: http://ssrn.com/abstract=1607759 (accessed 30 September 2016).

51. Mills T.C. and Jordanov J.V. (2000) Lead-lag patterns between small and large size portfolios in the London stock exchange. Applied Financial Economics, vol. 11, no. 5, pp. 489-495. doi:10.1080/096031001752236771.

52. Pastor L. and Stambaugh R.F. (2003) Liquidity risk and expected stock returns. The Journal of Political Economy, vol. 111, no. 3, pp. 642-685. doi:10.3386/w8462.

53. Reinganum M.R. (1981) Misspecification of capital asset pricing: Empirical anomalies based on earnings' yields and market values. Journal of Financial Economics, vol. 9, no. 1, pp. 19-46. doi:10.1016/0304-405X(81)90019-2.

54. Sehgal S. and TripathiV.(2005)Size effect in Indian stockmarket: some empirical evidence. The Journal of Business Perspective, vol. 9, no. 4, pp. 27-42. doi:10.1177/097226290500900403.

55. Sharpe W.F. (1964) Capital asset prices: A theory of market equilibrium under conditions of risk. The Journal of Finance, vol. 19, no. 3, pp. 425-442. doi:10.1111/j.1540-6261.1964. tb02865.x.

56. Shum W.C. and Tang G.Y. (2005) Common risk factors in returns in Asian emerging stock markets. International Business Review, vol. 14, pp. 695-717. doi:10.1016/j.ibusrev. 2005.09.001.

57. Singh R. (2009) Company attributes and stock returns in India: A panel data analysis. Journal of Applied Finance, vol. 15, pp. 46-57. Available at: http://crawl.prod.proquest. com.s3.amazonaws.com/fpcache/c76160f03383752cb6028b0a99dfaaa2.pdf?AWSAccessK eyId=AKIAJF7V7KNV2KKY2NUQ\&Expires $=1475435112 \&$ Signature $=$ FRGyv1SuN4m NDKLnmoIUWU47yj0\%3D (accessed 30 September 2016).

58. Tong W.H.S. (1992) An analysis of the January effect of the United States, Taiwan and South Korean stock returns. Asia Pacific Journal of Management, vol. 9, no. 2, pp. 189-207. doi:10.1007/BF01732896.

59. Wong K.A. and Lye M.S. (1990) Market values, earnings' yields and stock returnsevidence from Singapore. Journal of Banking and Finance, vol. 14, no. 2-3, pp. 311-326. 
doi:10.1016/0378-4266(90)90052-4.

60. Wu H. (2010) The Value and Size Effect-Are There Firm-Specific-Pisks in China's Domestic Stock Markets? International Journal of Economics and Finance, vol. 3, no. 3, pp. 26-37. doi:10.5539/ijef.v3n3p26.

61. Zhang X. F. (2006) Information uncertainty and stock returns. Journal of Finance, vol. 61, no. 1, pp. 105-136. doi:10.1111/j.1540-6261.2006.00831.x.

\title{
SIZE EFFECT AND COST OF EQUITY
}

\author{
Sofya Fomkina \\ Postgraduate Student, Faculty of Economic Sciences \\ Department of Finance / NRU HSE
}

\begin{abstract}
The size effect still remains one of the mysteries of capital markets. This effect was first discovered by Banz (Banz, 1981) during the test of asset pricing model (CAPM) in the US market. This discovery led to further investigation of the issue in other developed and developing capital markets. However, until now in the scientific community there is no consensus about the real presence of this effect in capital markets and its magnitude. At the same time, the size premium is actively used in the practice of companies, funds and individual analysts for evaluating the cost of equity

This paper presents an overview of studies devoted to analysis of the size effect on developed and developing capital markets. We systematized and summarized different approaches of assessing the size premiums, compared the obtained empirical results and summarized possible explanations for this effect.

In this study we also identified the features under the analysis of the size effect, discussed their causes, as well as the appearance of the effect, in general, in capital markets and its further disappearance in several countries.
\end{abstract}

Keywords: size effect, size premium, cost of equity, capital markets

JEL: G12, G15, G32

\section{Referens}

1. Aksu M.H. and Onder T. (2003) The Size and Book-to-Market Effects and Their Role as Risk Proxies in the Istanbul Stock Exchange. SSRN Working Paper. Available at: http://ssrn. com/abstract=250919 (accessed 30 September 2016).

2. Al-Mwalla M., Karasneh M. (2011) Fama \& French Three Factor Model: Evidence from Emerging Market. European Journal of Economics, Finance and Administrative Sciences, no. 41, pp. 132-141. Available at: http://s3.amazonaws.com/academia.edu. documents/32657095.

3. Al-Rjoub S.A.M., Varela O. and Hassan M.K. (2005) The size effect reversal in the USA. Applied Financial Economics, vol. 15, pp. 1189-1197. doi:10.1080/09603100500359542.

4. Amihud Y. (2002) Illiquidity and stock returns: cross-section and time-series effects. Journal of Financial Markets, vol. 5, no.1, pp. 31-56. doi:10.1016/S1386-4181(01)00024-6.

5. Amihud Y. and Mendelson H. (1986) Asset pricing and the bid-ask spread. Journal of Financial Economics, vol. 17, pp. 223-249. doi:10.1016/0304-405X(86)90065-6.

6. Annaert J., van Holle F., Crombez J. and Spinel B. (2002) Value and size effect: Now you see it, now you don't. SSRN Working Paper. Available at: http://ssrn.com/abstract=950899 (accessed 30 September 2016).

7. Apergis N. and Payne J.E. (2014) Resurrecting the size effect Evidence from a panel nonlinear 
cointegration model for the G7 stock markets. Review of Financial economics vol. 23, no.1, pp. 46-53. doi:10.1016/j.rfe.2013.08.003.

8. Banz R.W. (1981) The relationship between return and market value of common stocks. Journal of Financial Economics, vol. 9, no.1, pp. 3-18. doi:10.1016/0304-405X(81)90018-0.

9. Beedles W.L. (1992) Small firm equity cost: evidence from Australia. Journal of Small Business Management, vol. 30, pp. 57-65. Available at: http://crawl.prod.proquest. com.s3.amazonaws.com/fpcache/f00012e0d24400454a4ff2505169771a.pdf?AWSAccessK eyId=AKIAJF7V7KNV2KKY2NUQ\&Expires $=1475427763 \&$ Signature=KFXVBLkr\%2F gSqA1z4oPeri94cLKc\%3D (accessed 30 September 2016).

10. Bekaert G., Harvey C.R. and Lundbland C. (2007) Liquidity and expected returns: lessons from emerging markets. Review of Financial Studies, vol. 20, no6, pp. 1783-1831. doi:10.1093/rfs/hhm030

11. Berk B. (1996) An Empirical Re-examination of the Relation Between Firm Size and Return. Revised Working Paper 93-BJ-001 (1996), Seattle: School of Business Administration, University of Washington. Available at: http://citeseerx.ist.psu.edu/viewdoc/download? doi $=10.1 .1 .195 .7084 \& r e p=$ rep1\& type $=$ pdf $($ accessed 30 September 2016).

12. Black F., Jensen M.C. and Scholes M. (1972) The Capital Asset Pricing Model: Some Empirical Tests. SSRN Working Paper. Available at: http://ssrn.com/abstract $=908569$ (accessed 30 September 2016).

13. Brennan M.J. and Subrahmanyam A. (1996) Market microstructure and asset pricing: On the compensation for illiquidity in stock returns. Journal of Financial Economics, vol. 41, no. 3, pp. 441-464. doi:10.1016/0304-405X(95)00870-K.

14. Bundoo S.K. (2008) An Augmented Fama and French Three-Factor Model: New Evidence From An Emerging Stock Market. Applied Economics Letters, vol. 15, pp. 1213-1218. doi:10.1080/13504850601018049.

15. Chan L.K.C., Karceski J. and Lakonishok J. (2000) New paradigm or same old hype in equity investing? Financial Analysts Journal, vol. 56, no. 4, pp. 23-36. doi:10.2469/faj.v56. n4.2371.

16. Chen T.S. and Chien C.C. (2011) Size effect in January and cultural influences in an emerging stock market: The perspective of behavioral finance. Pacific-Basin Finance Journal, vol. 19, no. 2, pp. 208-229. doi:10.1016/j.pacfin.2010.10.002.

17. Chen Z., Ibbotson R.G. and Hu W.Y. (2010) Liquidity as an investment style. SSRN Working Paper. Available at: http://ssrn.com/abstract=1817889 (accessed 30 September 2016).

18. Cho S. (2012) The Size Premium: What Role Does Macroeconomic Risk Play? SSRN Working Paper. Available at: http://ssrn.com/abstract=1969828 (accessed 30 September 2016).

19. Daniel K. and Titman S. (1997) Evidence on the Characteristics of Cross Sectional variation in stock returns. Journal of Finance, vol. 52, no. 1, pp. 1-33. doi:10.1111/j.1540-6261. 1997.tb03806.x.

20. Dimson E. and Marsh P. (1999) Murphy's law and market anomalies. Journal of Portfolio Management, vol. 25, no. 2, 1999, pp. 53-69. Available at: http://ssrn.com/abstract $=137337$ (accessed 30 September 2016).

21. Doeswijk R.Q. (1997) Contrarian Investment in the Dutch Stock Market. De Economist, vol. 145, no. 4, pp. 573-598. doi:10.1023/A:1003133310376.

22. Drew M.E. and Veeraraghavan M. (2002) A closer look at the size and value premium in emerging markets: evidence from the Kuala Lumpur stock exchange. Asian Economic Journal, vol. 16, no. 4, pp. 337-351. doi:10.1111/1467-8381.00156.

23. Drew M.E. (2003) Beta, Firm Size, Book-to-Market Equity and Stock Returns: Further Evidence from Emerging Markets. Journal of the Asian Pacific Economy, vol. 8, no. 3, pp. 354-379. doi:10.1080/13547860306289. 
24. Drew M.E., Naughton T. and Veeraraghavan M. (2003) Firm size, book-to-market equity and security returns: evidence from the Shanghai Stock Exchange. Australian Journal of Management, vol. 28, no. 2, pp. 119-139. doi:10.1177/031289620302800201.

25. Easterday K.E., Sen P.K. and Stephan J.A. (2009) The persistence of the small firm/January effect: Is it consistent with investors' learning and arbitrage efforts? The Quarterly Review of Economics and Finance, vol. 49, no. 3, pp. 1172-1193. doi:10.1016/j.qref.2008.07.001.

26. Elfakhani S., Lockwood L.J. and Zaher T.S. (1998) Small Firm and Value Effects in the Canadian Stock Market. Journal of Financial Research, vol. 21, no. 3, pp. 277-292. doi:10.1111/j.1475-6803.1998.tb00686.x.

27. Eun C.S. and Huang W. (2007) Asset pricing in China's domestic stock markets: Is there a logic? Pacific-Basin Finance Journal, vol. 15, no. 5, pp. 52-480. doi:10.1016/j.pacfin. 2006.11.002

28. Fama E.F. and French K.R. (1992) The Cross-Section of Expected Stock Returns. The Journal of Finance, vol. 47, no. 2, pp. 427-465. doi:10.1111/j.1540-6261.1992.tb04398.x.

29. Fama E.F. and French K.R. (1993) Common risk factors in the returns on stocks and bonds. Journal of Financial Economics, vol.33, no. 1,pp. 3-56. doi:10.1016/0304-405X(93)90023-5.

30. Fama E.F., French K.R. (2012) Size, value, and momentum in international stock returns. Journal of Financial Economics, vol. 105, no 3, pp. 457-472. doi:10.1016/j.jfineco. 2012.05.011

31. Fama E.F. and MacBeth J.D. (1973) Risk, return and equilibrium: Empirical tests. Journal of Political Economy, vol. 81, no. 3, pp. 607-636. Available at: http://www.jstor.org/stable/ 1831028 ?seq=1\#page_scan_tab_contents (accessed 30 September 2016).

32. Garza-Gómez X., Hodoshima J. and Kunimura M. (1998) Does size really matter in Japan? Financial Analysts Journal, vol. 54, no. 6, pp. 22-34. doi:10.2469/faj.v54.n6.2222.

33. Gillan F.L. (1990) An Investigation into CAPM Anomalies in New Zealand: The Small Firm and Price-Earnings Ratio Effects. Asia Pacific Journal of Management, vol. 7, no. 2, pp. 63-78. doi:10.1007/BF01951479.

34. Grabowski R.J. (2013) Risk Premium Report. Chicago, Duff \& Phelps, LLC. Available at: http://www.duffandphelps.com/assets/pdfs/publications/valuation/(excerpt)\%202013 \%20duff\%20phelps\%20risk\%20premium\%20report.pdf (accessed 30 September 2016).

35. Hassan A. and Javed M.T. (2011) Size and value premium in Pakistani equity market. African Journal of Business Management, vol. 16, no. 5, pp. 6747-6755. doi:10.5897/AJBM10.817.

36. Hearn B., Piesse J. and Strange R. (2010) Market liquidity and stock size premia in emerging financial markets: the implications for foreign investment. International Business Review, vol. 19, no. 5, pp. 489-501. doi:10.1016/j.ibusrev.2009.02.009.

37. Herrera M.J. and Lockwood L.J. (1994) The Size Effect in the Mexican Stock Market. Journal of Banking and Finance, vol. 18, no. 4, pp. 621-632. doi:10.1016/0378-4266(93)00010-M/

38. Horowitz J.L., Loughran T. and Savin N.E. (2000a) The disappearing size effect. Research in Economics, vol. 54, no 1, pp. 83-100. doi:10.1006/reec.1999.0207.

39. Horowitz J.L., Loughran T., Savin N.E. (2000b) Three analyses of the firm size premium. Journal of Empirical Finance, vol. 7, no2, pp. 143-153. doi:10.1016/S0927-5398(00)00008-6

40. Ivashkovskaja I., Grigor'eva S., Kokoreva M., Stepanova A. i dr. (2012) Korporativnye finansovye reshenija. Ehmpiricheskijj analiz rossijjskikh kompanijj (korporativnye finansovye reshenija na razvivajushhikhsja rynkakh kapitala) [Corporate financial decisions. The empirical analysis of Russian companies (corporate finance solutions in emerging capital markets)]. Monografija [Monograph]. Pod nauch. red. d-ra ehkon. nauk I.V. Ivashkovskojj [Under the scientific editorship of Doctor of Economic Sciences I. Ivashkovskaya]. Moscow: INFRA-M (in Russian).

41. Keim D.B. (1983) Size-related anomalies and stock return seasonality: Further empirical 
evidence. Journal of Financial Economics, vol. 12, no. 1, pp. 13-32. doi:10.1016/0304405X(83)90025-9.

42. Karasneh

pdf?AWSAccessKeyId=

M._Fama_French_Three_Factor_Model_Evidence_f. 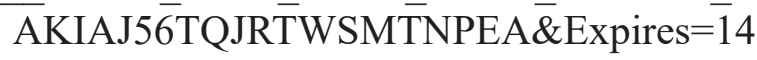

$75426899 \&$ Signature=n1QdBpYNktRiS a 1 NEXzYB L UvbZ4\%3 D \& r esponse-content-disposition=inline $\% 3 \mathrm{~B} \% 20$ filename $\% 3 \mathrm{DMahmoud}$ _Karasneh_Fama_and_French_Three_F.pdf(accessed 30 September 2016).

43. Khan F., Hassan A. and Ali S. (2012) Size, Leverage and stock returns: evidence from Pakistan. International Journal of Academic Research, vol. 4, no 1, pp. 24-32. Available at: http://s3.amazonaws.com/academia.edu.documents/34544274/SIZE LEVERAGE AND_STOCKS_RETURNS_EVIDENCE.pdf?AWSAccessKeyId=AKIAJ56TQJRTW SMTNNPEA\&Expires $=1475430530 \&$ Signature $=$ RVnosG7JD1bJ5vzFMs6RJItjZuY\%3 D\&response-content-disposition $=$ inline $\% 3 B \% 20$ filename\%3D24_PART_B._SOCIAL_ SCIENCES_AND_HUMANITIE.pdf (accessed 30 September 2016).

44. Lakonishok J. and Shapiro A.C. (1986) Systematic risk, total risk and size as determinants of stock market returns. Journal of Banking and Finance, vol. 10, no. 1, pp. 115-132. doi:10.1016/0378-4266(86)90023-3.

45. Lee K. (2011) The world price of liquidity risk. Journal of Financial Economics, vol. 99, no. 1, pp. 136-161. doi:10.1016/j.jineco.2010.08.003.

46. Leledakis G., Davidson I. and Karathanassis G. (2003) Cross-sectional estimation of stock returns in small markets: The case of the Athens Stock Exchange. Applied Financial Economics, vol. 13, no. 6, pp. 413-426. doi:10.1080/09603100210143118.

47. Lemmon M. and Portniaguina E. (2006) Consumer confidence and asset prices: Some empirical evidence. The Review of Financial Studies, vol. 19, no. 4, pp. 1499-1529. doi:10.1093/rfs/hhj038.

48. Lintner J. (1965) The Valuation of Risk Assets and the Selection of Risky Investments in Stock Portfolios and Capital Budgets. The Review of Economics and Statistics, vol. 47, no. 1, pp. 13-37. doi:10.2307/1924119.

49. Lo A.W. and MacKinlay A.C. (1990) Data-snooping biases in tests of financial asset pricing models. Review of Financial Studies, vol. 3, no. 3, pp. 431-467. doi:10.1093/rfs/3.3.431.

50. Michou M., Mouselli S. and StarkA. (2010) Fundamental analysis and the modelling of normal returns in the UK. SSRN Working Paper. Available at: http://ssrn.com/abstract=1607759 (accessed 30 September 2016).

51. Mills T.C. and Jordanov J.V. (2000) Lead-lag patterns between small and large size portfolios in the London stock exchange. Applied Financial Economics, vol. 11, no. 5, pp. 489-495. doi:10.1080/096031001752236771.

52. Pastor L. and Stambaugh R.F. (2003) Liquidity risk and expected stock returns. The Journal of Political Economy, vol. 111, no. 3, pp. 642-685. doi:10.3386/w8462.

53. Reinganum M.R. (1981) Misspecification of capital asset pricing: Empirical anomalies based on earnings' yields and market values. Journal of Financial Economics, vol. 9, no. 1, pp. 19-46. doi:10.1016/0304-405X(81)90019-2.

54. Sehgal S. andTripathiV.(2005)Size effect in Indian stockmarket: someempirical evidence. The Journal of Business Perspective, vol. 9, no. 4, pp. 27-42. doi:10.1177/097226290500900403.

55. Sharpe W.F. (1964) Capital asset prices: A theory of market equilibrium under conditions of risk. The Journal of Finance, vol. 19, no. 3, pp. 425-442. doi:10.1111/j.1540-6261.1964. tb02865.x.

56. Shum W.C. and Tang G.Y. (2005) Common risk factors in returns in Asian emerging stock markets. International Business Review, vol. 14, pp. 695-717. doi:10.1016/j.ibusrev. 2005.09.001. 
57. Singh R. (2009) Company attributes and stock returns in India: A panel data analysis. Journal of Applied Finance, vol. 15, pp. 46-57. Available at: http://crawl.prod.proquest. com.s3.amazonaws.com/fpcache/c76160f03383752cb6028b0a99dfaaa2.pdf?AWSAccessK eyId=AKIAJF7V7KNV2KKY2NUQ\&Expires=1475435112\&Signature=FRGyv1SuN4m NDKLnmoIUWU47yj0\%3D (accessed 30 September 2016).

58. Tong W.H.S. (1992) An analysis of the January effect of the United States, Taiwan and South Korean stock returns. Asia Pacific Journal of Management, vol. 9, no. 2, pp. 189-207. doi:10.1007/BF01732896.

59. Wong K.A. and Lye M.S. (1990) Market values, earnings' yields and stock returnsevidence from Singapore. Journal of Banking and Finance, vol. 14, no. 2-3, pp. 311-326. doi:10.1016/0378-4266(90)90052-4.

60. Wu H. (2010) The Value and Size Effect -Are There Firm-Specific-Pisks in China's Domestic Stock Markets? International Journal of Economics and Finance, vol. 3, no. 3, pp. 26-37. doi:10.5539/ijef.v3n3p26.

61. Zhang X. F. (2006) Information uncertainty and stock returns. Journal of Finance, vol. 61, no. 1, pp. 105-136. doi:10.1111/j.1540-6261.2006.00831.x.

\section{ДИВИДЕНДНЫЕ ВЫПЛАТЫ РОССИЙСКИХ КОМПАНИЙ В УСЛОВИЯХ ФИНАНСОВОГО КРИЗИСА}

\section{Анкудинов Андрей Борисович} Лебедев Олег Владимирович²

В работе приведены результаты эмпирического анализа динамики дивидендных выплат отечественных компаний в условиях кредитного сжатия кризисного периода. Анализ основан на панельных данных; выборка сформирована по данным крупнейших российских компаний нефинансового сектора за период 2003-2011 гг. Метод исследования состоял из двух частей: сначала был проведен одномерный анализ зависимости индивидуальных характеристик компаний и проводимой ими дивидендной политики, затем был проведен многовариантный анализ на основе регрессионного оценивания по панельным данным. Полученные оценки показывают, что сформировавшиеся к настоящему времени подходы к распределению прибыли российских компаний таковы, что многие из них не выплачивают дивиденды вовсе (66\% наблюдений относительно долей распределенной на дивиденды прибыли представляют нулевые значения). В среднем доля прибыли, направляемая на дивиденды, составляла 8,5\% (25\% для подвыборки компаний - плательщиков дивидендов), что ниже дивидендного выхода компаний как с развитых, так и со многих развивающихся рынков, и характеризовалась высоким разбросом в пространственном и временном измерении. Четко идентифицируется снижение дивидендов выплат в кризисные годы. В предкризисный период государственные и частные компании характеризовались примерно равной величиной дивидендного выхода, однако в посткризисный период частные компании распределяли на дивиденды большую часть прибыли. Разница в величине выплаченных дивидендов публичных и непубличных компаний в условиях финансового кризиса снижается, однако первые характеризуются существенно более щедрой дивидендной политикой. Ненаблюдаемые индивидуальные характеристики компании оказывают существенное влияние на величину распределяемой на дивиденды прибыли. Прибыльность, размер компании позитивно, а финансовый леверидж негативно коррелированы с вероятностью выплаты каких-либо дивидендов и с величиной выплачиваемых дивидендов. Позитивная зависимость прибыльности и доли прибыли, распределяемой на дивиденды, усиливается в посткризисный период. В то же время негативная

\footnotetext{
${ }^{1}$ Кандидат физико-математических наук, доцент кафедры финансов организации, Казанский федеральный университет, научный сотрудник Университета Иннополис, e-mail: ankudia@mail.ru

${ }^{2}$ Ассистент кафедры финансов организации, Казанский федеральный университет, научный сотрудник Университета Иннополис, e-mail: lebolegan@yandex.ru
} 